\title{
Acoustic source localization in anisotropic plates without knowing their material properties: an experimental investigation
}

Novonil Sen, Mateusz Gawroński, Pawel Packo, Tadeusz Uhl, Tribikram Kundu

Novonil Sen, Mateusz Gawroński, Pawel Packo, Tadeusz Uhl, Tribikram Kundu, "Acoustic source localization in anisotropic plates without knowing their material properties: an experimental investigation," Proc. SPIE 10972, Health Monitoring of Structural and Biological Systems XIII, 1097224 (1 April 2019); doi: 10.1117/12.2513467

Event: SPIE Smart Structures + Nondestructive Evaluation, 2019, Denver, Colorado, United States 


\title{
Acoustic source localization in anisotropic plates without knowing their material properties: an experimental investigation
}

\author{
Novonil Sen*a, Mateusz Gawroński ${ }^{\mathrm{b}}$, Pawel Packo ${ }^{\mathrm{b}}$, Tadeusz Uhl ${ }^{\mathrm{b}}$ and Tribikram Kundu ${ }^{\mathrm{a}, \mathrm{c}}$ \\ aDepartment of Aerospace and Mechanical Engineering, University of Arizona, Tucson, AZ 85721, \\ USA; ${ }^{b}$ AGH University of Science and Technology, Krakow, Poland; 'Department of Civil and \\ Architectural Engineering and Mechanics, University of Arizona, Tucson, AZ 85721, USA
}

\begin{abstract}
An integral aspect of modern infrastructural engineering is to constantly monitor the health of a structure either actively or passively in order to ensure its safe performance throughout the design life. For passive structural health monitoring, it is important to estimate the location of an acoustic source that may be caused by events such as impact of a foreign object with the structure, failure of a structural element, formation of cracks, etc. Such an acoustic source generates acoustic waves that propagate through the medium. These waves can be captured by ultrasonic sensors mounted on the structure at some pre-selected locations and, subsequently, analyzed to predict the location of the acoustic source. Over the years, several researchers have proposed techniques for acoustic source localization in both isotropic and anisotropic structures. While acoustic source localization in isotropic structures is relatively simple, introduction of anisotropy adds a layer of difficulty to the problem due to the fact that waves do not propagate with the same speed in all directions. This study presents acoustic source localization techniques for anisotropic plates based on the analysis of the wave front shapes typically observed in anisotropic plates and presents experimental verification of the techniques. Three different geometric shapes are considered as the assumed wave front shapes: a rhombus, an ellipse and a parametric curve. A slightly modified version of the rhombus-based technique from the original approach is proposed. The experimental study is performed on two plates with different degrees of anisotropy.
\end{abstract}

Keywords: Passive structural health monitoring, acoustic source, ultrasonic sensors, acoustic source localization, anisotropic structures, wave front shapes, experimental verification

\section{INTRODUCTION}

Safe and intended performance of a structure can be ensured by constantly monitoring its current health to detect and mitigate any type of damage occurring during its design life. This process, commonly known as structural health monitoring (SHM) in literature, can be carried out either via active or passive techniques. For passive SHM, it is important to estimate, as accurately as possible, the potential damage initiation location in the structure as a first step of the diagnosis stage. Typically, various events such as impact of a foreign object with the structure, failure of a structural element, formation of cracks, etc. can potentially lead to or indicate damage at the location of these events. These events, commonly known as acoustic events, generate acoustical waves that propagate from the source through the structure. These wave-signals can be recorded by several ultrasonic sensors installed at some specific locations of the structure, and then the recorded signals can be analyzed to predict the source location by employing suitable techniques, commonly called acoustic source localization (ASL) techniques. There have been several studies in the past proposing ASL techniques in isotropic structures ${ }^{1-13}$. However, most of the modern structures are made of anisotropic materials. ASL in anisotropic structures is typically more challenging than that in isotropic structures owing to the fact that acoustic waves propagate energy in a direction-dependent pattern in an anisotropic material. Development of ASL techniques in such anisotropic structures has received attention only in the recent past. The beamforming method, which was previously applicable to isotropic plates only ${ }^{3,4}$, was improved by Nakatani et al. ${ }^{14-15}$ to make it applicable to anisotropic plates. In their method, the expression of the beamforming array was modified by taking the direction-dependent wave speed into account. Kundu et al. ${ }^{16,17}$ proposed an ASL technique in anisotropic plates which is based on the minimization of an objective function containing the time difference of arrival of waves at the sensors and direction-dependent speed

* Corresponding author.

E-mail addresses: novonilsen@email.arizona.edu (N. Sen), matgawr@agh.edu.pl (M. Gawroński), pawel.packo@agh.edu.pl (P. Packo), tuhl@agh.edu.pl (T. Uhl), tkundu@email.arizona.edu (T. Kundu)

Health Monitoring of Structural and Biological Systems XIII, edited by Paul Fromme,

Zhongqing Su, Proc. of SPIE Vol. 10972, 1097224 - (c) 2019 SPIE

CCC code: $0277-786 \mathrm{X} / 19 / \$ 18 \cdot$ doi: $10.1117 / 12.2513467$ 
information. This approach was further modified by Hajzargerbashi et al. ${ }^{18}$ to improve the ASL accuracy considering a new objective function that simplifies the optimization process. For anisotropic plates with unknown material properties, Kundu ${ }^{19}$ and Kundu et al. ${ }^{20}$ proposed a technique which performs ASL by acquiring and analyzing signal data at several sensors mounted on a plate in right-angled L-shaped clusters. Their method requires sufficiently accurate estimation of the time difference of arrival (TDOA) of waves at the sensors of a cluster. This TDOA estimation was carried out by cross-correlating the signals. This concept was extended by Yin et al. ${ }^{21}$ and Z-shaped clusters were introduced. Their method requires less sensors than those in Kundu ${ }^{19}$ and Kundu et al. ${ }^{20}$ to achieve the similar degree of ASL accuracy. Ciampa et al. ${ }^{22,23}$ proposed a technique for ASL in anisotropic plates with unknown material properties. Their method estimates the time of arrival (TOA) using continuous wavelet transform, and requires a nonlinear problem to be solved to estimate the source location. Kundu et al. ${ }^{24}$ proposed a technique that uses the source location obtained by the method in Kundu et al. ${ }^{20}$ as an initial guess for minimizing an objective function to arrive at a more accurate prediction than the initial guess. A linearized algorithm was proposed by Simone et al. ${ }^{25}$ for ASL in a composite plate using four sensors. Sabzevari and Moavenian ${ }^{26}$ proposed an attenuation analysis-based technique which also uses four sensors. Xiao et al. ${ }^{27}$ proposed an ASL technique by using beamforming approach with two arrays of sensors placed in mutually perpendicular directions. No a priori knowledge of material properties is needed in their technique. Jang and $\mathrm{Kim}^{28}$ extended the triangulation method ${ }^{1}$, commonly applicable to isotropic plates, to be applicable to anisotropic plates by using neural network training.

Most of the studies discussed so far assume that acoustic waves propagate along a straight line path from the acoustic source to a sensor, which is an unrealistic assumption for anisotropic materials ${ }^{29}$. Baxter et al..$^{30}$ considered a complex structure where due to complexity in the structural configuration waves are unable to follow a straight line path. This technique requires the structure to be trained by a set of training data. Their technique was modified by Hensman et al. ${ }^{31}$ such that less amount of training data is needed. Gollob et al. ${ }^{32}$ proposed an ASL technique based on a modified Dijkstra algorithm. Although their approach does not make the assumption of the straight line wave propagation, it needs the knowledge of the direction-dependent wave speed. Park et al. ${ }^{33}$ proposed a wave front shape-based approach to ASL in anisotropic plates with unknown material properties, where no assumption of the straight line wave propagation is made. Their method assumes different non-circular geometric shapes (namely, rhombus and ellipse) as the shapes of the wave fronts, and predicts the source location by analyzing those shapes and incorporating the signal data. Sen and Kundu ${ }^{34}$ modified the elliptical wave front-based method proposed by Park et al..$^{33}$ to properly relate the wave front geometry with the recorded signal data, and also proposed a more general parametric curve-based approach.

In Park et al. ${ }^{33}$ and Sen and Kundu ${ }^{34}$, the proposed ASL techniques were verified through numerical simulations. In the present study, the rhombus ${ }^{33}$, elliptical ${ }^{34}$ and parametric curve ${ }^{34}$-based techniques are considered and their effectiveness in ASL for anisotropic plates is studied through experimental investigations. Two sample plates with different degrees of anisotropy are considered for this purpose. The rhombus-based technique has been slightly modified in this paper from the original approach in Park et al. ${ }^{33}$ in order to obtain the "shape speed" more realistically.

The wave front shape-based approaches mentioned in the previous paragraph use the right-angled L-shaped sensor cluster configuration proposed by Kundu ${ }^{19}$ and Kundu et al. ${ }^{20}$. In the following sections, this configuration is discussed first, and the estimation method of the angle of arrival of the signal at a sensor cluster is described. Following this, the rhombus $^{33}$, elliptical ${ }^{34}$ and parametric curve ${ }^{34}$-based techniques are described. Finally, the experimental setup and the wave front shape-based ASL results obtained in two anisotropic plates with different degrees of anisotropy are discussed.

\section{SENSOR CLUSTER CONFIGURATION AND INCIDENCE ANGLE ESTIMATION}

Fig. 1 shows the L-shaped sensor cluster configuration (originally proposed in Kundu ${ }^{19}$ and Kundu et al. ${ }^{20}$ ) used in this paper. For the experimental verification of the wave front-shape based approaches, this study considers the sensor clusters to be located with respect to the acoustic source such that the waves arrive at different clusters from different directions as shown in Figs. 1(a)-1(c). In these figures, the black dots represent three sensors $A, O$ and $B$ in a cluster. To distinguish between the three directions of arrival, the corresponding sensor clusters are referred to as Type-a, Type-b and Type-c clusters, respectively, in this paper (it may be observed that Type-b sensor cluster of the present study is identical to Type-a sensor cluster of Sen and $\left.K u_{n d u}^{34}\right)$. The single-headed arrows indicate the wave propagation directions. The sensor clusters are mounted on an anisotropic plate such that the lines $A O$ and $O B$ are parallel to the $x$ and 
$y$-axes of the coordinate system, respectively. The distance between $A$ and $O$, denoted by $d$, is same as that between $O$ and $B$. The value of $d$ should be sufficiently small so that the signal features do not vary significantly among the three sensors in a cluster. Also, $d$ needs to be much smaller than the distance between the acoustic source and a sensor cluster ${ }^{33}$. Since $d$ is small, it may be assumed that although the material is anisotropic, the wave fronts passing through a sensor cluster are planar (shown in Fig. 1 as parallel lines perpendicular to the wave propagation directions) and propagate with same speed $v$ while striking all the three sensors in a cluster. It may be noted that $v$ is expected to be different at different sensor clusters due to anisotropy. Also, the coordinates of any of the sensors $A, O$ or $B$ in a cluster may be regarded as the coordinates of that cluster, since the three sensors are closely spaced. In this study, the coordinates of $O$ are considered as the cluster coordinates. The incidence angle for a wave acquired at a sensor cluster is denoted by $\theta$. The TOAs at sensors $A, O$ and $B$ are denoted by $t_{A}, t_{O}$ and $t_{B}$, respectively. For Type-a sensor cluster (see Fig. 1(a)), the signal arrives at either sensor $A$ or sensor $B$ first, and at sensor $O$ in the end, i.e., $t_{O}>t_{A}$ and $t_{O}>t_{B}$. Thus, for Type-a sensor cluster, we may write

$$
\tan \alpha=\frac{\sin \alpha}{\cos \alpha}=\frac{v\left(t_{O}-t_{B}\right) / d}{v\left(t_{O}-t_{A}\right) / d}=\frac{t_{O}-t_{B}}{t_{O}-t_{A}}=\frac{t_{B}-t_{O}}{t_{A}-t_{O}}
$$

Therefore,

$$
\alpha=\tan ^{-1}\left(\frac{t_{B}-t_{O}}{t_{A}-t_{O}}\right)
$$

and,

$$
\theta=\alpha+\pi=\tan ^{-1}\left(\frac{t_{B}-t_{O}}{t_{A}-t_{O}}\right)+\pi
$$

Similarly, for the Type-b sensor cluster, the signal arrives at sensor $O$ first, and then strikes either $A$ or $B$, i.e., $t_{A}>t_{O}$ and $t_{B}>t_{O}$. Thus, for Type-b sensor cluster, we may write

$$
\tan \theta=\frac{\sin \theta}{\cos \theta}=\frac{v\left(t_{B}-t_{O}\right) / d}{v\left(t_{A}-t_{O}\right) / d}=\frac{t_{B}-t_{O}}{t_{A}-t_{O}}
$$

Therefore,

$$
\theta=\tan ^{-1}\left(\frac{t_{B}-t_{O}}{t_{A^{-}-t_{O}}}\right)
$$

Lastly, for the Type-c sensor cluster, the signal arrives at sensor $A$ first, then strikes sensor $O$ and finally strikes sensor $B$, i.e., $t_{B}>t_{O}>t_{A}$. Thus, for Type-c sensor cluster, we may write

$$
\tan \beta=\frac{\sin \beta}{\cos \beta}=\frac{v\left(t_{B}-t_{O}\right) / d}{v\left(t_{O}-t_{A}\right) / d}=\frac{t_{B}-t_{O}}{t_{O}-t_{A}}
$$

Therefore,

$$
\beta=\tan ^{-1}\left(\frac{t_{B}-t_{O}}{t_{O}-t_{A}}\right)
$$

So,

$$
\theta=\pi-\beta=\pi-\tan ^{-1}\left(\frac{t_{B}-t_{O}}{t_{O}-t_{A}}\right)=\tan ^{-1}\left(\frac{t_{B}-t_{O}}{t_{A}-t_{O}}\right)+\pi
$$

From Eq. (3) and (8), it may be observed that $\theta$ is given by the same expression for Type-a and Type-c sensor clusters.

Since the accuracy of the estimation of $\theta$ is largely dictated by the accuracy of the estimated values of $t_{A}, t_{O}$ and $t_{B}$, these TOAs need to be obtained as accurately as possible. For this purpose, Akaike Information Criterion $(A I C)^{12,29,34}$ is used in this study. AIC corresponding to a time $t$ is given by 


$$
A I C(k)=k \ln [\operatorname{var}\{U(1: k)\}]+(K-k-1) \ln [\operatorname{var}\{U(k+1: K)\}]
$$

where $k$ is the data point number corresponding to $t$ in the recorded signal data at a sensor, $K$ is the total number of data points, $U$ is the set of recorded signal amplitude data points and $\operatorname{var}\{U(i: j)\}$ is the variance of the signal amplitude data points between and including the $i$ th and the $j$ th points. By varying $k$ from 1 to $(K-1)$, AIC for all the signal data points can be computed from Eq. (9), which can then be plotted against $t$. The plot ideally shows several distinct valleys. The first and second valleys represent the arrivals of the first (corresponding to the first symmetric mode) and second (corresponding to the first antisymmetric mode) groups of acoustic waves, respectively. Thus, the values of $t$ corresponding to the first and second valleys are the required TOAs of the first and second groups of waves, respectively.

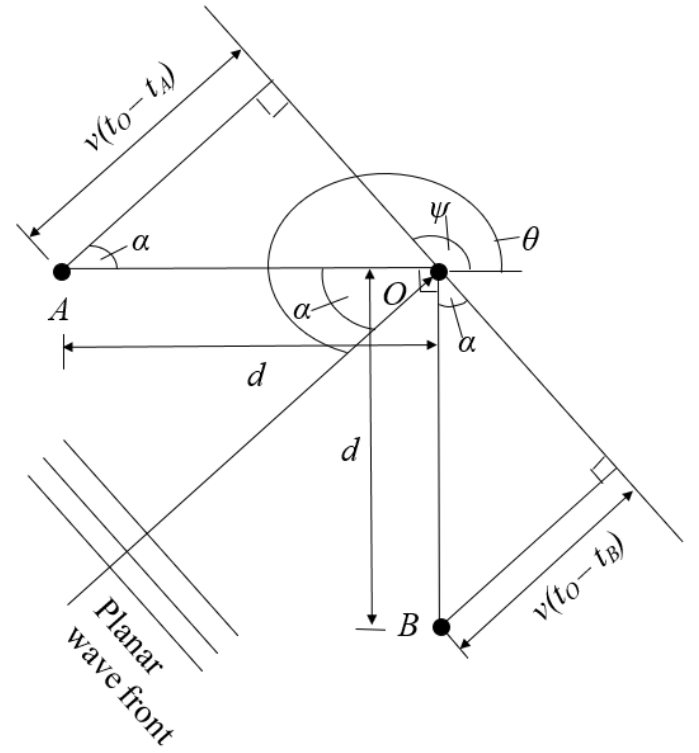

(a)

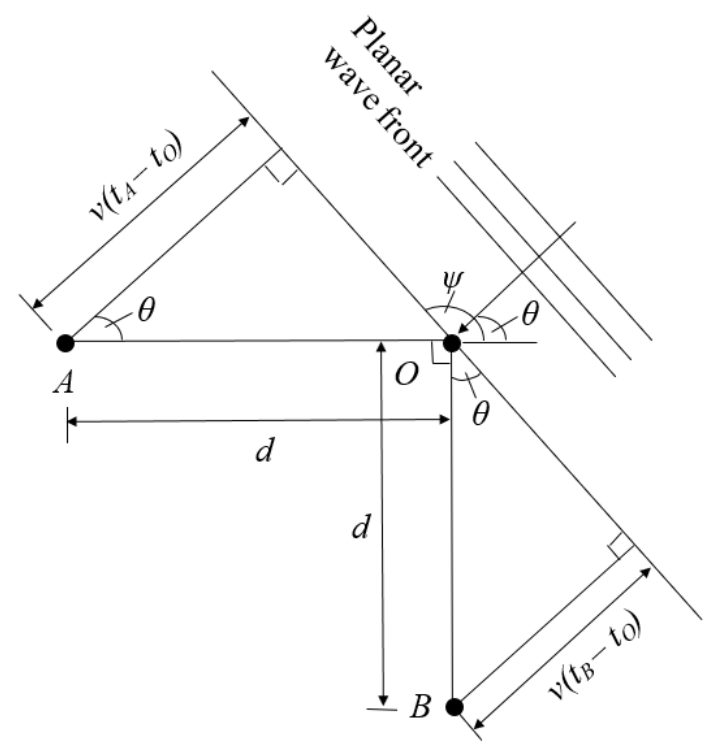

(b)

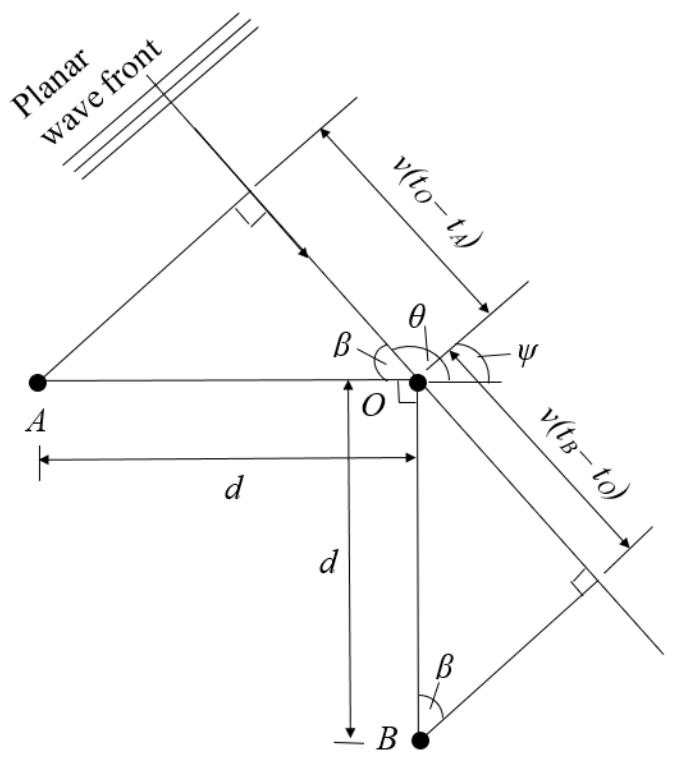

(c)

Figure 1. (a) Type-a, (b) Type-b and (c) Type-c sensor clusters. 


\section{WAVE FRONT SHAPE-BASED TECHNIQUES}

\subsection{Rhombus wave front shape-based technique}

Park et al. ${ }^{33}$ observed via a numerical study that the first group of waves generated due to an acoustic event propagates in an anisotropic plate in such a fashion that the resulting wave fronts resemble the geometric shape of a rhombus. Therefore, the shape of the first group wave fronts may be idealized as rhombus. Fig. 2 shows a schematic diagram of the rhombus wave fronts, the acoustic source and the three sensor clusters I, II and III. It may be noted that, although the original approach proposed in Park et al.$^{33}$ requires four sensor clusters, the modified version of the rhombus wave frontshape based approach requires three sensor clusters, as will be discussed later in this sub-section. Sensor clusters $I, I I$ and III are of Type-a, Type-b and Type-c, respectively (see Section 2). Three concentric rhombuses passing through each sensor cluster are shown in this figure. The intersecting point of the two diagonals $L_{1} L_{2}$ and $L_{3} L_{4}$ of the rhombuses is the location of the acoustic source. The $x$ and $y$ axes of the coordinate system are not necessarily parallel to the two diagonals.

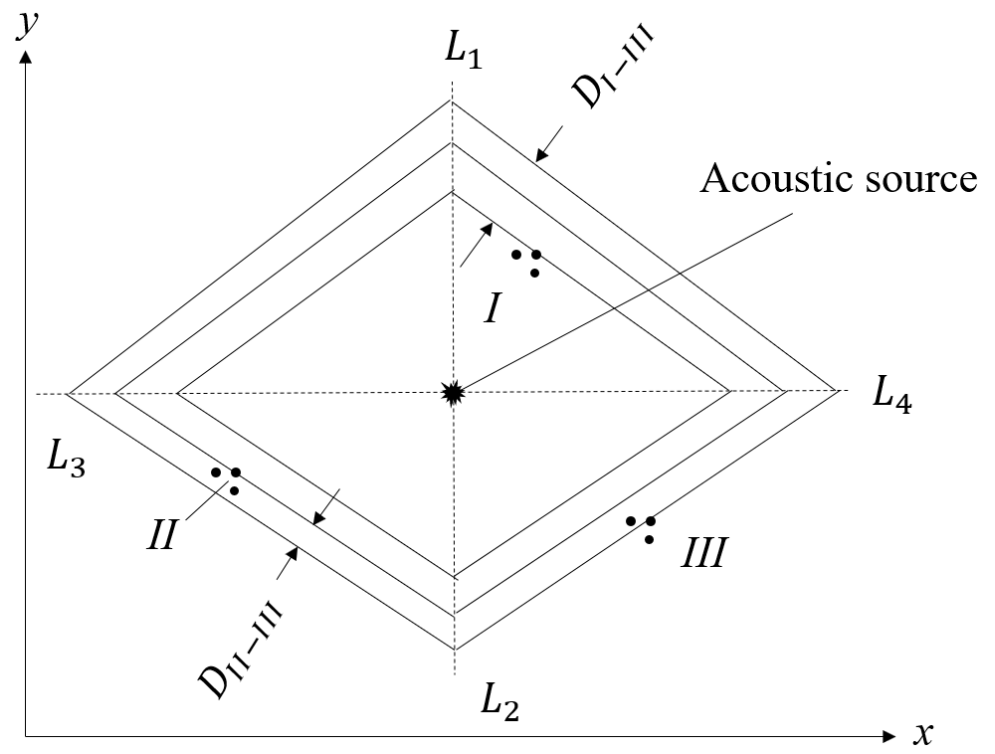

Figure 2. Schematic diagram of rhombus wave fronts, the acoustic source and sensor clusters.

The angles of incidence $\theta$ of the waves at the sensor clusters can be estimated using Eqs. (3), (5) and (8) for sensor clusters $I, I I$ and $I I I$, respectively. Let $\mathbf{u}$ be the unit vector along the wave propagation direction (see Fig. 1 for the propagation direction) at a cluster, and $\mathbf{v}$ be the unit vector along the direction that is $90^{\circ}$ clockwise with the direction of $\mathbf{u}$ (i.e., $\mathbf{v}$ lies along the planar wave front in Fig. 1). Thus, these unit vectors can be computed as:

$$
\mathbf{u}=\left[\begin{array}{ll}
-\cos \theta & -\sin \theta
\end{array}\right]^{T}
$$

and

$$
\mathbf{v}=\left[\begin{array}{ll}
-\sin \theta & \cos \theta
\end{array}\right]^{T}
$$

The speed $(\mu)$ of the rhombus wave front is required to be estimated, which was proposed to be performed in Park et al. ${ }^{33}$ by installing another sensor cluster on the plate in addition to the clusters $I, I I$ and $I I I$. This extra sensor cluster was proposed to be installed in Park et al..$^{33}$ at the same quadrant (out of the four quadrants created by the two rhombus diagonals) where cluster $I$ is mounted, and then $\mu$ was proposed to be estimated by dividing the distance between cluster $I$ and the extra cluster along the wave propagation direction by the TDOA between these two clusters. However, 
estimating this distance can be erroneous, especially when cluster $I$ and the extra cluster are located far apart. To avoid this, in the present study, the extra sensor cluster is eliminated and $\mu$ is proposed to be estimated simply by taking the average value of the wave speeds at the clusters $I, I I$ and III. From Fig. 1 and Eqs. (1)-(8), it follows that the wave speed at a sensor cluster can be determined from either of the following two equations:

$$
\begin{aligned}
& v=\left|d \sin \theta /\left(t_{B}-t_{O}\right)\right| \\
& v=\left|d \cos \theta /\left(t_{A}-t_{O}\right)\right|
\end{aligned}
$$

Therefore,

$$
\mu=\frac{v_{I}+v_{I I}+v_{I I I}}{3}
$$

where $v_{I}, v_{I I}$ and $v_{I I I I}$ are the wave speeds calculated using Eq. (12) or (13) at clusters $I, I I$ and $I I I$, respectively.

As shown in Fig. 2, a single rhombus wave front will most likely not pass through the three clusters at the same time instant. Let us consider clusters $I I$ and $I I I$. Let us say that the waves strike cluster $I I$ first and then cluster $I I I$. So, at the time instant when a wave front reaches cluster $I I I$, the wave front has already passed through and shifted from cluster $I I$. This shift occurs in the direction of $\mathbf{u}_{I I}$, where $\mathbf{u}_{I I}$ is the unit vector $\mathbf{u}$ at cluster $I I$. It may be noted from Fig. 2 that the shifted line forms the third quadrant side of the rhombus passing through cluster III. The magnitude of the shift (shown in Fig. 2) can be determined as

$$
D_{I I-I I I}=\mu\left(T_{I I I}-T_{I I}\right)
$$

where $T_{I I}$ and $T_{I I I}$ are the TOAs at clusters $I I$ and $I I I$, respectively. In this study, $T_{I I}$ and $T_{I I I}$ are same as $t_{O}$ at clusters $I I$ and $I I I$, respectively. Therefore, the position vector $\mathbf{p}_{1}$ of any point located on the third quadrant side of the rhombus passing through cluster $I I I$ is given by

$$
\mathbf{p}_{1}=\mathbf{s}_{I I}+D_{I I-I I I} \mathbf{u}_{I I}+\kappa_{1} \mathbf{v}_{I I}
$$

where $\mathbf{s}_{I I}$ is the position vector of cluster $I I, \kappa_{1}$ is a real number and $\mathbf{v}_{I I}$ is the unit vector $\mathbf{v}$ at cluster $I I$. At the same time instant, we can express the position vector $\mathbf{p}_{2}$ of any point located on the fourth quadrant side of the rhombus passing through cluster $I I I$ as

$$
\mathbf{p}_{2}=\mathbf{s}_{I I I}+\kappa_{2} \mathbf{v}_{I I I}
$$

where $\mathbf{s}_{I I I}$ is the position vector of cluster $I I I, \kappa_{2}$ is a real number and $\mathbf{v}_{I I I}$ is the unit vector $\mathbf{v}$ at cluster $I I I$. Now, the position vector $\mathbf{p}_{3}$ of the intersecting point of the third and fourth quadrant sides of the rhombus passing through cluster III can be found by equating $\mathbf{p}_{1}$ and $\mathbf{p}_{2}$ and solving for $\kappa_{1}$ and $\kappa_{2}$. This operation yields

$$
\left[\begin{array}{ll}
\kappa_{1} & \kappa_{2}
\end{array}\right]^{T}=\left[\begin{array}{ll}
\mathbf{v}_{I I} & -\mathbf{v}_{I I I}
\end{array}\right]^{-1}\left(\mathbf{s}_{I I I}-\mathbf{s}_{I I}-D_{I I-I I I} \mathbf{u}_{I I}\right)
$$

Thus, $\mathbf{p}_{3}$ can be found by substituting $\kappa_{1}$ and $\kappa_{2}$ obtained using Eq. (18) into either Eq. (16) or (17). Next, the position vector $\mathbf{p}_{4}$ of any point on the rhombus diagonal $L_{1} L_{2}$ can be determined as

$$
\mathbf{p}_{4}=\mathbf{p}_{3}+\kappa_{3} \mathbf{b}_{1}
$$

where $\kappa_{3}$ is a real number and $\mathbf{b}_{1}$ is a vector along $L_{1} L_{2}$. Since the diagonal $L_{1} L_{2}$ bisects the rhombus angle at the intersecting point (i.e., the point for which the position vector is $\mathbf{p}_{3}$ ) into two equal angles, $\mathbf{b}_{1}$ can be expressed as

$$
\mathbf{b}_{1}=\mathbf{u}_{I I}+\mathbf{u}_{I I I}
$$

where $\mathbf{u}_{I I I}$ is the unit vector $\mathbf{u}$ at cluster $I I I$. It may be noted that although it has been assumed in the beginning of this paragraph that $T_{I I I}>T_{I I}$, the above derivations remain valid even when $T_{I I I}<T_{I I}$, in which case $D_{I I-I I I}$ given by Eq. (15) will simply be negative and the rest of the computations up to this point will remain same.

In the similar manner, we can also analyze the other diagonal $L_{3} L_{4}$. Considering clusters $I$ and $I I I$, let us say that the waves strike cluster $I$ first and then cluster $I I I$. At the time instant when a wave front reaches cluster $I I I$, the wave front has already passed through and shifted from cluster $I$. This shift occurs in the direction of $\mathbf{u}_{I}$, where $\mathbf{u}_{I}$ is the unit vector $\mathbf{u}$ at cluster $I$. It may be noted from Fig. 2 that the shifted line forms the first quadrant side of the rhombus passing through cluster III. The magnitude of the shift (shown in Fig. 2) can be determined as

$$
D_{I-I I I}=\mu\left(T_{I I I}-T_{I}\right)
$$


where $T_{I}$ is the TOA at cluster $I$. In this study, $T_{I}$ is same as $t_{O}$ at cluster $I$. Therefore, the position vector $\mathbf{p}_{5}$ of any point located on the first quadrant side of the rhombus passing through cluster III is given by

$$
\mathbf{p}_{5}=\mathbf{s}_{I}+D_{I-I I I} \mathbf{u}_{I}+\kappa_{4} \mathbf{v}_{I}
$$

where $\mathbf{s}_{I}$ is the position vector of cluster $I, \kappa_{4}$ is a real number and $\mathbf{v}_{I}$ is the unit vector $\mathbf{v}$ at cluster $I$. Now, the position vector $\mathbf{p}_{6}$ of the intersecting point of the first and fourth quadrant sides of the rhombus passing through cluster III can be found by equating $\mathbf{p}_{5}$ and $\mathbf{p}_{2}$ and solving for $\kappa_{2}$ and $\kappa_{4}$. This operation yields

$$
\left[\begin{array}{ll}
\kappa_{4} & \kappa_{2}
\end{array}\right]^{T}=\left[\begin{array}{ll}
\mathbf{v}_{I} & -\mathbf{v}_{I I I}
\end{array}\right]^{-1}\left(\mathbf{s}_{I I I}-\mathbf{s}_{I}-D_{I-I I I} \mathbf{u}_{I}\right)
$$

It should be noted that $\kappa_{2}$ obtained from Eq. (23) is not necessarily the same as that obtained from Eq. (18). Now, $\mathbf{p}_{6}$ can be found by substituting $\kappa_{4}$ and $\kappa_{2}$ obtained using Eq. (23) into either Eq. (17) or (22). Next, the position vector $\mathbf{p}_{7}$ of any point on the rhombus diagonal $L_{3} L_{4}$ can be determined as

$$
\mathbf{p}_{7}=\mathbf{p}_{6}+\kappa_{5} \mathbf{b}_{2}
$$

where $\kappa_{5}$ is a real number and $\mathbf{b}_{2}$ is a vector along $L_{3} L_{4}$. Since the diagonal $L_{3} L_{4}$ bisects the rhombus angle at the intersecting point (i.e., the point for which the position vector is $\mathbf{p}_{6}$ ) into two equal angles, $\mathbf{b}_{2}$ can be expressed as

$$
\mathbf{b}_{2}=\mathbf{u}_{I}+\mathbf{u}_{I I I}
$$

It may be noted that although it has been assumed in the beginning of this paragraph that $T_{I I I}>T_{I}$, the above derivations remain valid even when $T_{I I I}<T_{I}$, in which case $D_{I-I I I}$ given by Eq. (21) will simply be negative and the rest of the computations up to this point will remain same.

The position vector $\mathbf{p}_{\text {source }}$ of the intersecting point of $L_{1} L_{2}$ and $L_{3} L_{4}$ (i.e., the acoustic source) can now be evaluated by equating $\mathbf{p}_{4}$ and $\mathbf{p}_{7}$ and solving for $\kappa_{3}$ and $\kappa_{5}$. This operation yields

$$
\left[\begin{array}{ll}
\kappa_{3} & \kappa_{5}
\end{array}\right]^{T}=\left[\begin{array}{ll}
\mathbf{b}_{1} & -\mathbf{b}_{2}
\end{array}\right]^{-1}\left(\mathbf{p}_{6}-\mathbf{p}_{3}\right)
$$

$\mathbf{p}_{\text {source }}$ can then be found by substituting $\kappa_{3}$ and $\kappa_{5}$ obtained from Eq. (26) into either Eq. (19) or (24). Let the acoustic source coordinates be $\left(C_{x}, C_{y}\right)$. Thus,

$$
\left[\begin{array}{ll}
C_{x} & C_{y}
\end{array}\right]^{T}=\mathbf{p}_{\text {source }}
$$

\subsection{Elliptical wave front shape-based technique}

Park et al ${ }^{33}$ observed that the second group of waves generated due to an acoustic event propagates in an anisotropic plate in such a manner that the resulting wave front shape can be idealized as an ellipse. An ASL technique based on the elliptical wave fronts was proposed in Park et al. ${ }^{33}$, which was later modified by Sen and Kundu ${ }^{34}$ to properly relate the wave front geometry with the recorded signal data. This sub-section describes the elliptical wave front-shape based technique proposed in Sen and Kundu ${ }^{34}$.

Fig. 3 shows a schematic diagram of an elliptical wave front and the acoustic source. The equation of the elliptical wave front is given by

$$
\frac{\left(x-C_{x}\right)^{2}}{a^{2}}+\frac{\left(y-C_{y}\right)^{2}}{b^{2}}=1
$$

where $(x, y)$ are the coordinates of a point on the ellipse, and $a$ and $b$ are the lengths of the semi-major and semi-minor axes, respectively. Taking derivative of Eq. (28) with respect to $x$ yields

$$
m=-\frac{b^{2}}{a^{2}} \frac{x-C_{x}}{y-C_{y}}
$$

where $m=\frac{d y}{d x}$. Since the wave fronts are almost concentric ${ }^{33}, 34, \frac{b^{2}}{a^{2}}$ may be replaced by a constant $\lambda$. Thus, we may write from Eq. (29):

$$
\lambda\left(x-C_{x}\right)+m\left(y-C_{y}\right)=0
$$

Now, the wave fronts pass through the sensor clusters installed. Thus, for $i$ th sensor cluster, we can write from Eq. (30): 


$$
\lambda\left(x_{i}-C_{x}\right)+m_{i}\left(y_{i}-C_{y}\right)=0
$$

where $\left(x_{i}, y_{i}\right)$ are the coordinates of the $i$ th sensor cluster and $m_{i}$ is $m$ for the $i$ th cluster. From Fig. 1 , it may be observed that

$$
m_{i}=\tan \psi_{i}
$$

where $\psi_{i}$ is the angle $\psi$ (see Fig. 1) for the $i$ th cluster. Since $\psi=\theta-\pi / 2$ (for Type-a and Type-c clusters) or $\theta+\pi / 2$ (for Type-b cluster), it follows from Eq. (32) that

$$
m_{i}=-\frac{1}{\tan \theta_{i}}
$$

where $\tan \theta_{i}$ is $\tan \theta$ for the $i$ th cluster which can be obtained as (see Section 2)

$$
\tan \theta=\frac{t_{B}-t_{O}}{t_{A}-t_{O}}
$$

for all three types of sensor clusters. Eq. (31) contains three unknowns: $\lambda, C_{x}$ and $C_{y}$. If a total of $n$ sensor clusters are considered, Eq. (31) gives $n$ simultaneous equations containing these three unknowns. To evaluate the unknowns in least-squares sense, the following residual $\Phi$ can be minimized:

$$
\Phi\left(\lambda, C_{x}, C_{y}\right)=\sum_{i=1}^{n}\left[\lambda\left(x_{i}-C_{x}\right)+m_{i}\left(y_{i}-C_{y}\right)\right]^{2}
$$

The minimization of $\Phi$ given by Eq. (35) can be carried out using Levenberg-Marquardt algorithm or any other unconstrained optimization technique. The reader is referred to Sen and Kundu ${ }^{34}$ for the solution technique of the unknowns (that include the acoustic source coordinates) using Levenberg-Marquardt algorithm.

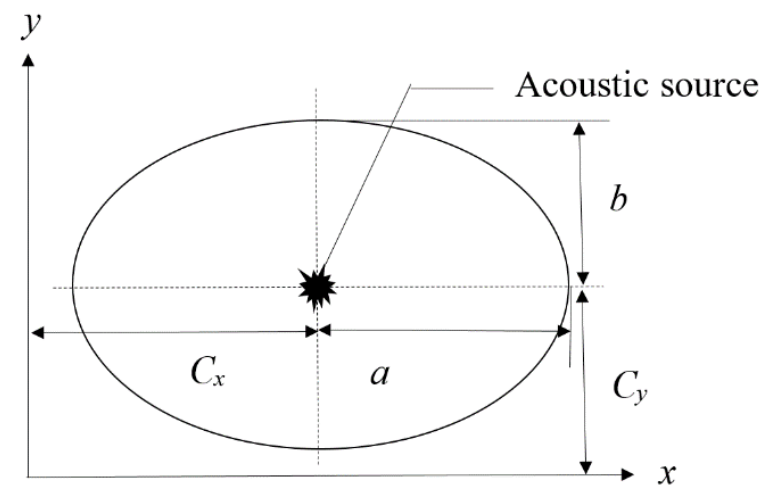

Figure 3. Schematic diagram of an elliptical wave front and the acoustic source (adapted from Sen and $\mathrm{Kundu}^{34}$ ).

\subsection{Parametric curve-based technique}

Sen and Kundu ${ }^{34}$ observed that the second group of waves propagates in an anisotropic plate in such a way that the resulting wave fronts almost look elliptical, although they may deviate from an exact elliptical shape. In order to make the elliptical shape more general, Sen and Kundu ${ }^{34}$ proposed a parametric curve to be considered as the wave front shape. The expression of such a parametric curve is given by

$$
\gamma \frac{\left|x-C_{x}\right|^{p+1}}{p+1}+\frac{\left|y-C_{y}\right|^{q+1}}{q+1}=c
$$

where $(x, y)$ are the coordinates of a point on the parametric curve, $\gamma, p$ and $q$ are three unknown positive numbers and $c$ is an arbitrary constant. Taking derivative of Eq. (36) with respect to $x$ yields

$$
m=(-1)^{\text {quad }} \gamma \frac{\left|x-C_{x}\right|^{p}}{\left|y-C_{y}\right|^{q}}
$$


with quad denoting the quadrant number of the point $(x, y)$ with respect to the straight lines $x=C_{x}$ and $y=C_{y}$, i.e.,

$$
\begin{aligned}
\text { quad } & =1 \quad \text { if } x>C_{x} \text { and } y \geq C_{y} \\
& =2 \quad \text { if } x \leq C_{x} \text { and } y>C_{y} \\
& =3 \quad \text { if } x<C_{x} \text { and } y \leq C_{y} \\
& =4 \text { if } x \geq C_{x} \text { and } y<C_{y}
\end{aligned}
$$

It may be observed from Eq. (36) that the parametric curve becomes an ellipse when $p=q=1$, i.e., an ellipse is a special case of a parametric curve. For $i$ th sensor cluster, we can re-write Eq. (37) as

$$
(-1)^{\text {quad }_{i}} \gamma\left|x_{i}-C_{x}\right|^{p}-m_{i}\left|y_{i}-C_{y}\right|^{q}=0
$$

where quad $_{i}$ is quad for the $i$ th sensor cluster. Eq. (39) contains five unknowns: $p, q, \gamma, C_{x}$ and $C_{y}$. If a total of $n$ sensor clusters are considered, Eq. (39) gives $n$ simultaneous equations containing these five unknowns. To evaluate the unknowns in least-squares sense, the following residual $S$ can be minimized:

$$
S\left(p, q, \gamma, C_{x}, C_{y}\right)=\sum_{i=1}^{n}\left[(-1)^{\text {quad }_{i}} \gamma\left|x_{i}-C_{x}\right|^{p}-m_{i}\left|y_{i}-C_{y}\right|^{q}\right]^{2}
$$

To make the optimization procedure simpler, $q$ may be held constant at a convenient value, i.e., $q$ is no longer an unknown and the residual may be re-expressed as

$$
S\left(p, \gamma, C_{x}, C_{y}\right)=\sum_{i=1}^{n}\left[(-1)^{q^{q u a d}} \gamma\left|x_{i}-C_{x}\right|^{p}-m_{i}\left|y_{i}-C_{y}\right|^{q}\right]^{2}
$$

Sen and $\mathrm{Kundu}^{34}$ set $q=1$. The same is used in this study. The minimization of $S$ given by Eq. (41) can be carried out using a simplex algorithm, or any other unconstrained optimization technique. The reader is referred to Sen and Kundu ${ }^{34}$ for the solution technique of the unknowns (that include the acoustic source coordinates) using Nelder-Mead simplex algorithm ${ }^{35,36}$.

\section{EXPERIMENTAL STUDY}

The wave front shape-based techniques for ASL discussed in Section 3 were verified by Park et al. ${ }^{33}$ and Sen and $\mathrm{Kundu}^{34}$ through numerical illustrations. It is also desirable to verify the effectiveness of this methodology experimentally so as to know its suitability in real life applications. For this purpose, an acoustic event is generated in two anisotropic plates with different degrees of anisotropy and the acoustic source location is predicted using the presented approach. Then the predicted source coordinates are compared with the actual coordinates to investigate the effectiveness of the ASL approach.

In the following sub-sections, the experimental setup is discussed first, followed by the discussion on the ASL results.

\subsection{Experimental setup}

Two anisotropic plates are considered for the experimental verification of the wave front shape-based techniques. The first plate, denoted by Plate 1 in this study, is a symmetric $\left(0^{\circ} / 45^{\circ} / 90^{\circ}\right)$ composite plate with carbon fiber reinforcement, processed by temperature shock. The dimension of Plate 1 is $800 \mathrm{~mm} \times 260 \mathrm{~mm} \times 2 \mathrm{~mm}$. Fig. 4 shows a schematic diagram of Plate 1 along with the coordinate system considered, the locations of the sensor clusters $I, I I$ and $I I I$, and the acoustic source location. The coordinates of the sensors are listed in Table 1. 


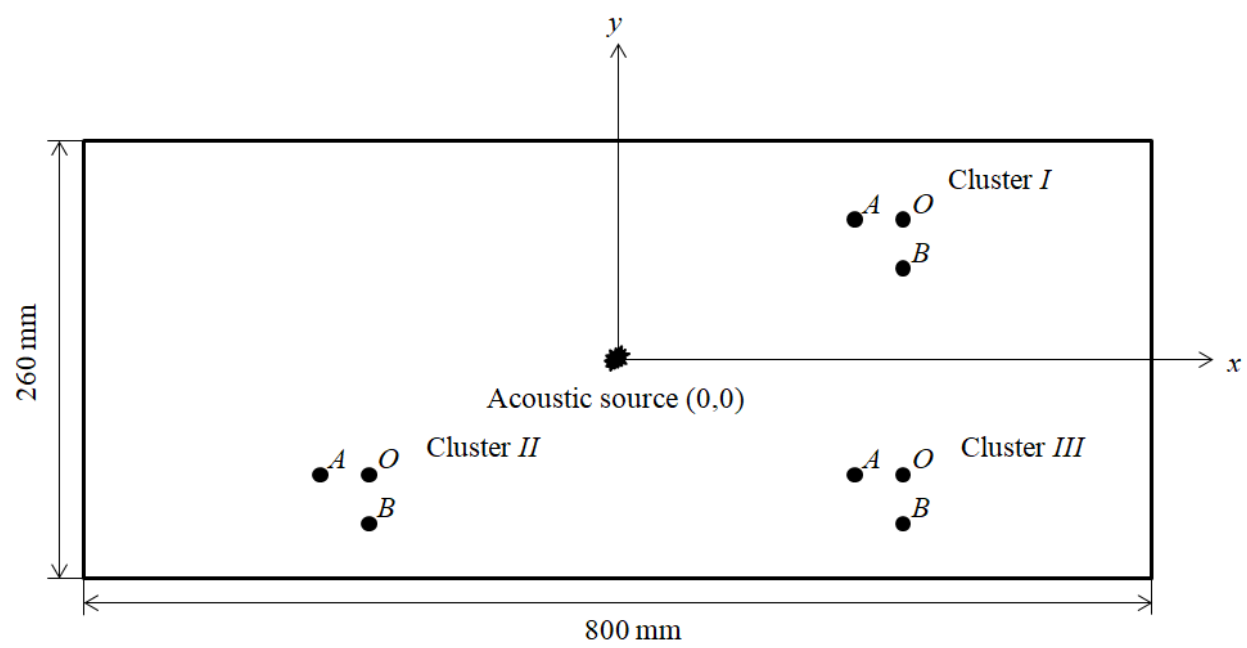

Figure 4. Schematic diagram of Plate 1 with coordinate system, sensor cluster locations and acoustic source location shown.

The second plate, denoted by Plate 2 in this study, is a pre-preg carbon Hexply composite plate, with a dimension of 500 $\mathrm{mm} \times 500 \mathrm{~mm} \times 4 \mathrm{~mm}$. Fig. 5 shows a schematic diagram of Plate 2 along with the coordinate system considered, the locations of the sensor clusters $I, I I$ and $I I I$, and the acoustic source location. The coordinates of the sensors are listed in Table 1.

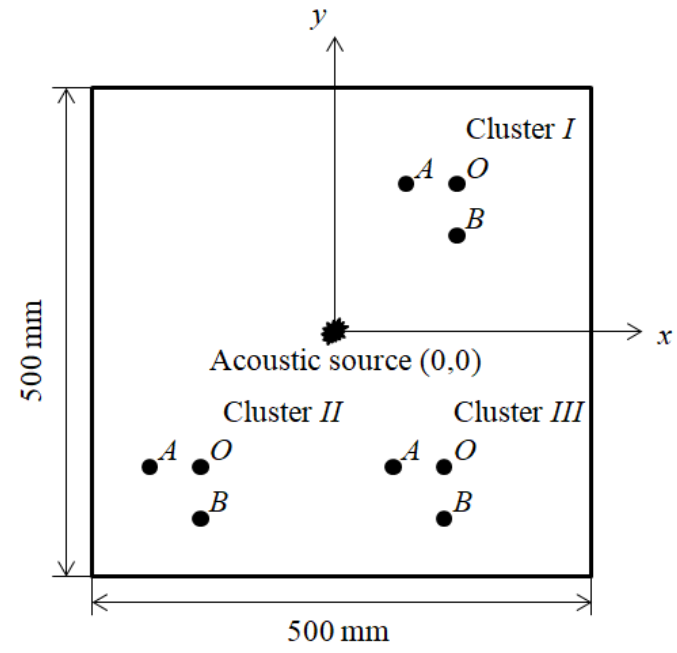

Figure 5. Schematic diagram of Plate 2 with coordinate system, sensor cluster locations and acoustic source location shown. 
Table 1. The coordinates of the sensors in both plates.

\begin{tabular}{|c|c|c|c|c|c|}
\hline Cluster & Sensor & \multicolumn{2}{|c|}{ Plate 1} & \multicolumn{2}{c|}{ Plate 2 } \\
\cline { 2 - 6 } & & $x(\mathrm{~mm})$ & $y(\mathrm{~mm})$ & $x(\mathrm{~mm})$ & $y(\mathrm{~mm})$ \\
\hline \multirow{3}{*}{$I$} & $A$ & 180 & 105 & 100 & 130 \\
\cline { 2 - 6 } & $O$ & 210 & 105 & 130 & 130 \\
\cline { 2 - 6 } & $B$ & 210 & 75 & 130 & 100 \\
\hline \multirow{3}{*}{$I I$} & $A$ & -210 & -75 & -160 & -130 \\
\cline { 2 - 6 } & $O$ & -180 & -75 & -130 & -130 \\
\cline { 2 - 6 } & $B$ & -180 & -105 & -130 & -160 \\
\hline \multirow{3}{*}{$I I I$} & $A$ & 180 & -75 & 100 & -130 \\
\cline { 2 - 6 } & $O$ & 210 & -75 & 130 & -130 \\
\cline { 2 - 6 } & $B$ & 210 & -105 & 130 & -160 \\
\hline
\end{tabular}

The ultrasonic sensors that are used in both plates belong to Vallen AMSY-6 system equipped with piezoelectric transducers (see Fig. 6) with operating frequency of $100-450 \mathrm{kHz}$ and resonant frequency of $150 \mathrm{kHz}$ with external preamplifiers. The distance $(d)$ between sensors $A$ and $O$, and $O$ and $B$ (see Fig. 1) is considered as $30 \mathrm{~mm}$ (as can be seen from Table 1) which is sufficiently small, satisfying the presented ASL approach requirement. For both plates, the signal at each sensor is recorded at a sampling rate of $5 \mathrm{MHz}$. Total 8192 and 1024 signal data points are captured at each sensor for Plate 1 and Plate 2, respectively. The acoustic sources at the center of both plates are generated by HSU pencil-lead break tests. Since the center is considered as the origin of the coordinate system for both plates (see Figs. 4 and 5), the actual coordinates of the acoustic sources are $(0,0)$ in both plates.

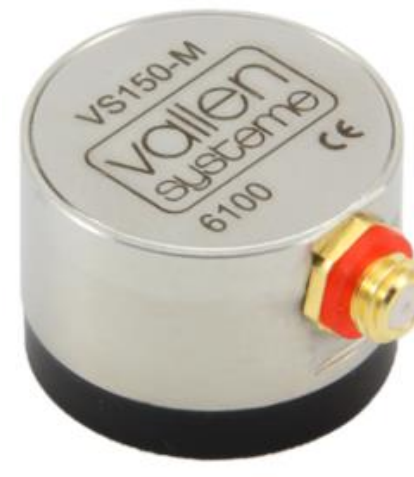

(a)

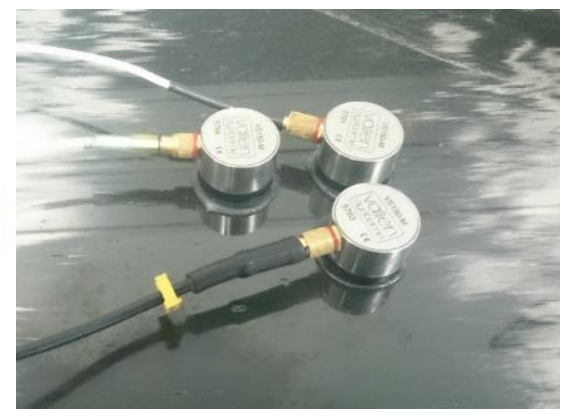

(b)

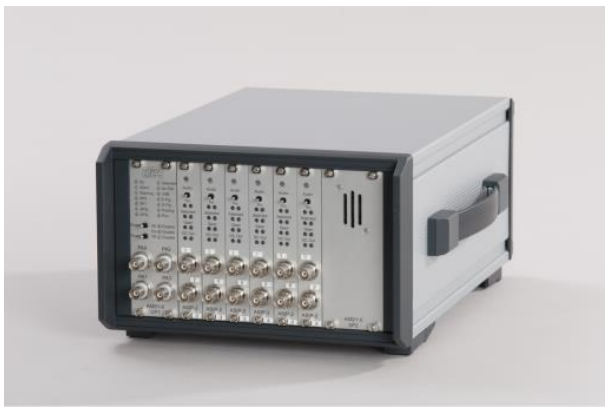

(c)

Figure 6. (a) A sensor, (b) a sensor cluster mounted on a plate and (c) Vallen AMSY-6 system equipped with piezoelectric transducers.

\subsection{Acoustic source localization results}

For sensors $O$ (see Fig. 1) in clusters $I, I I$ and $I I I$ in both plates, the recorded time histories of the signal amplitudes and the corresponding AIC plots are shown in Figs. 7 and 8. For Plate 1, the first 1500 points in the recorded signal data are used in this study for all sensors and the same is done for showing Fig. 7., whereas for Plate 2, all the recorded points (i.e., 1024) in the signal data are used. It may be observed from Figs. 7 and 8 that the first valley is distinct in the AIC plot for all the sensors (marked by a circle in an AIC plot) and the corresponding time is the TOA of the first wave groups at the sensor under consideration (see Section 2). However, the second valley is not obvious in the AIC plots in most of the cases. Therefore, although ideally the TOAs of the second wave groups should be used for elliptical and parametric curve-based approaches, in this study the TOAs of the first wave groups will be used for all three techniques described in Section 3. The similar plots as in Figs. 7 and 8 for the other sensors (i.e., $A$ and $B$ ) are not shown in this paper since the above discussion is also valid for them. 

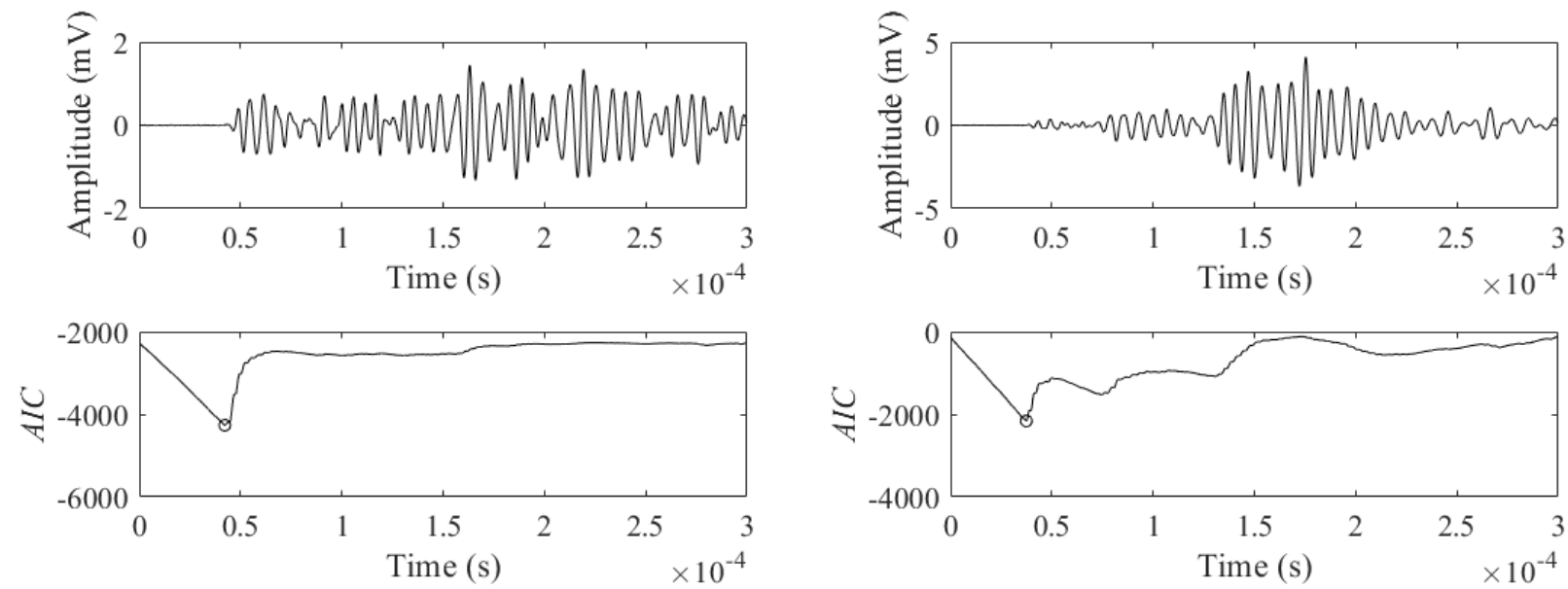

(a)

(b)
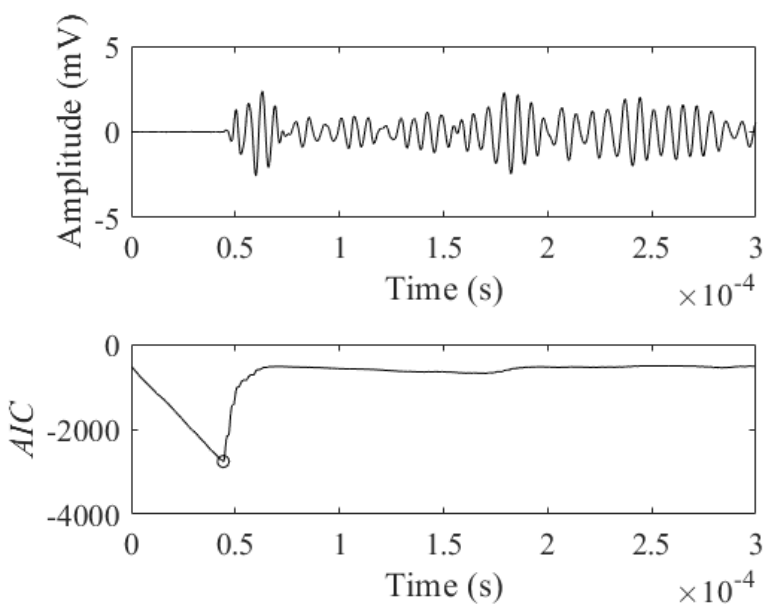

(c)

Figure 7. Time history and the corresponding $A I C$ plot of signal amplitudes recorded at sensor $O$ of (a) cluster $I$, (b) cluster $I I$ and (c) cluster III for Plate 1.

Proc. of SPIE Vol. 10972 1097224-12 

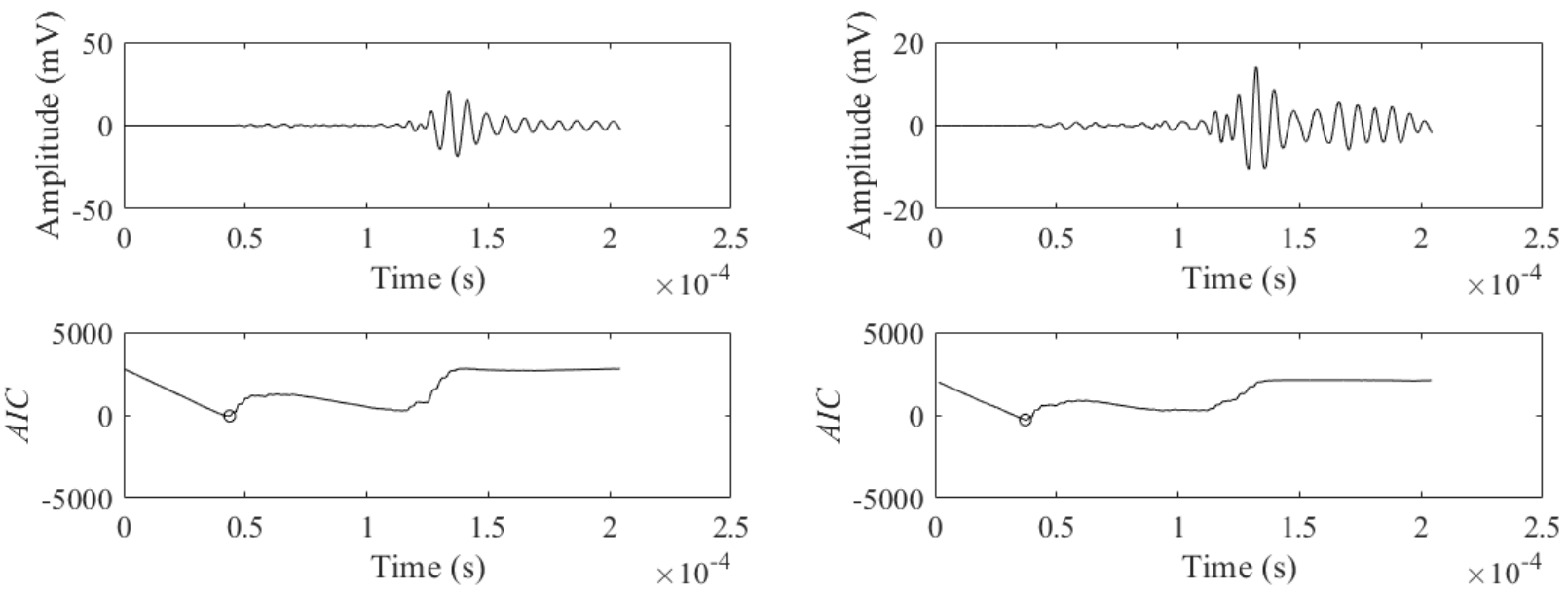

(a)

(b)
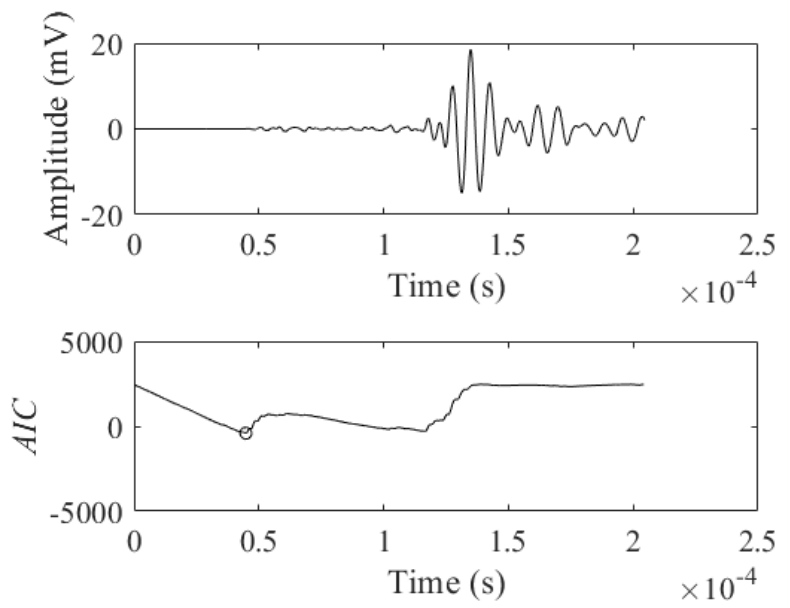

(c)

Figure 8. Time history and the corresponding AIC plot of signal amplitudes recorded at sensor $O$ of (a) cluster $I$, (b) cluster $I I$ and (c) cluster III for Plate 2 .

The location of the acoustic source is predicted by employing the techniques described in Section 3 and these predicted locations are listed in Table 2 for both plates, along with the errors $\varepsilon$ in the source localization, given by

$$
\varepsilon=\sqrt{\left(C_{x}-C_{x, \text { actual }}\right)^{2}+\left(C_{y}-C_{y, \text { actual }}\right)^{2}}
$$

where $\left(C_{x, \text { actual }}, C_{y, \text { actual }}\right)$ are the actual source coordinates (i.e., $(0,0)$ in this study). 
Table 2. Source localization results.

\begin{tabular}{|c|c|c|c|c|c|c|}
\hline \multirow{2}{*}{$\begin{array}{c}\text { ASL } \\
\text { Technique }\end{array}$} & \multicolumn{3}{|c|}{ Plate 1 } & \multicolumn{3}{c|}{ Plate 2 } \\
\cline { 2 - 7 } & $C_{x}(\mathrm{~mm})$ & $C_{y}(\mathrm{~mm})$ & $\varepsilon(\mathrm{mm})$ & $C_{x}(\mathrm{~mm})$ & $C_{y}(\mathrm{~mm})$ & $\varepsilon(\mathrm{mm})$ \\
\hline Rhombus & 13.23 & 36.59 & 38.91 & -26.10 & 8.04 & 27.31 \\
\hline Ellipse & -40.33 & 1.50 & 40.35 & -31.36 & -41.79 & 52.24 \\
\hline Parametric & -34.53 & 1.50 & 34.56 & -26.45 & -41.79 & 49.45 \\
\hline
\end{tabular}

The source localization results are also shown graphically in Figs. 9 and 10 for Plate 1 and Plate 2, respectively. In these two figures, 'rhombus' represents the rhombus wave front passing through sensor cluster III; 'prop dir I', 'prop dir II' and 'prop dir $I I I$ ' are the directions of wave propagation at clusters $I, I I$ and $I I I$, respectively (i.e., the directions of $\mathbf{u}_{I}$, $\mathbf{u}_{I I}$ and $\mathbf{u}_{I I I}$, respectively); 'actual' is the actual source location; 'rhombus pred', 'ellipse pred' and 'param pred' are the source locations predicted using rhombus-based, ellipse-based and parametric curve-based techniques, respectively; 'ellp thr $I$ ', 'ellp thr $I I$ ' and 'ellp thr $I I I$ ' are the elliptical wave fronts passing through sensor clusters $I, I I$ and $I I I$, respectively; 'param thr $I$ ', 'param thr $I I$ ' and 'param thr $I I I$ ' are the parametric curve wave fronts passing through sensor clusters $I, I I$ and $I I I$, respectively; and 'plate dimen' is the dimension of the plate. It may be noted that since the angle $\theta$ at cluster $I$ is not same as that at cluster $I I$, the first and third quadrant sides of the rhombus are not exactly parallel, and hence the rhombus is not a "true" rhombus. Similarly, the second and fourth quadrant sides of the rhombus are not parallel. 


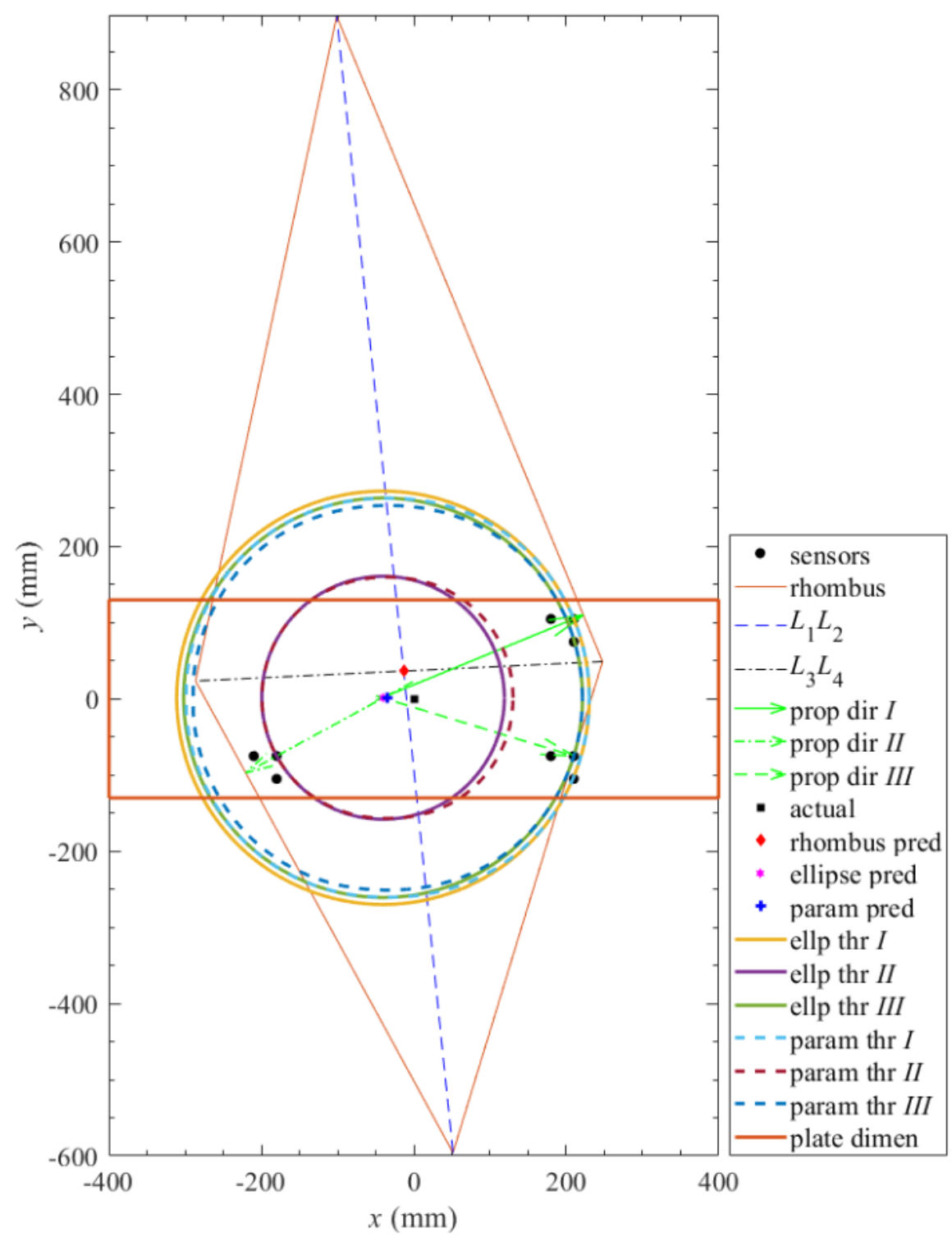

Figure 9. Source localization results for Plate 1. 


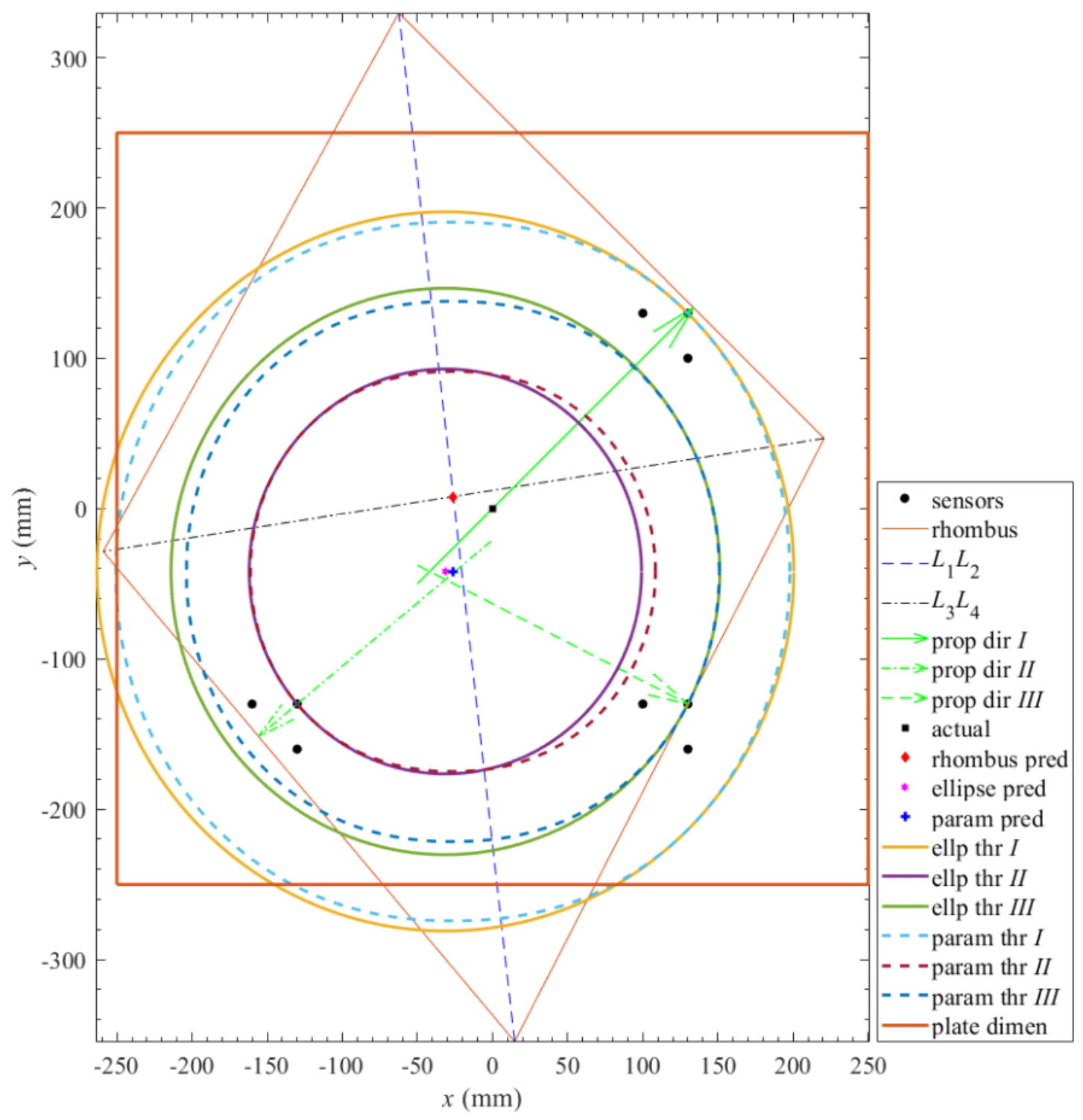

Figure 10. Source localization results for Plate 2.

From Table 2 and Figs. 9 and 10, it follows that the prediction errors lie within $60 \mathrm{~mm}$ in all the cases considered. While the parametric curve-based approach gives the best source prediction in Plate 1, the best ASL result is obtained in Plate 2 with the rhombus-based approach. The ellipse and parametric curve-based techniques predict acoustic source locations that lie quite close to each other, with parametric curve-based technique giving slightly better results. It may be noted that since there are four unknowns in the parametric curve-based technique and in the numerical study three sensor clusters are used, the source prediction is sensitive to the choice of the initial vertices, i.e., the ASL result will change (not significantly, however) if a different set of initial vertices in the simplex algorithm ${ }^{34}$ is used than what has been used in this paper (listed in Table 3). The source coordinates in the initial vertices in Table 3 are in the units of "mm". 
Table 3. Five initial vertices for both plates (in the form $\left[\begin{array}{llll}p & \gamma & C_{x} & C_{y}\end{array}\right]^{T}$ ).

\begin{tabular}{|c|c|c|c|c|c|}
\hline \multicolumn{3}{|c|}{ Plate 1} & \multicolumn{3}{|c|}{ Plate 2} \\
\hline$[1.1$ & 0.5 & $\left.\begin{array}{ll}-40.1 & 0.1\end{array}\right]^{T}$ & {$[1.1$} & 0.5 & $-40.1 \quad 0.1]^{T}$ \\
\hline$[1.2$ & 1.2 & $0.1 \quad-30.1]^{T}$ & {$[0.8$} & 1.2 & $\left.0.1{ }^{0}-30.1\right]^{T}$ \\
\hline$[1.2$ & 0.5 & $\left.90.1{ }^{-70.1}\right]^{T}$ & {$[1.2$} & 0.5 & $90.1-70.1]^{T}$ \\
\hline$[1.2$ & 0.7 & $\left.10.1{ }^{-18.1}\right]^{T}$ & {$[1.4$} & 0.3 & $10.1-18.1]^{T}$ \\
\hline$[1.8$ & 0.2 & $60.1 \quad 80.1]^{T}$ & {$[1.8$} & 0.2 & $60.1 \quad 80.1]^{T}$ \\
\hline
\end{tabular}

From Fig. 9, the ellipses and the parametric curves almost resemble circles, whereas Fig. 10 shows that they deviate significantly from circular shapes. This indicates that the degree of anisotropy in Plate 2 is higher than that in Plate 1. Also, it may be observed that in Plate 1, the assumption of waves propagating in straight lines leads to almost same order of source prediction errors as obtained by the ellipse and parametric curve-based techniques, since the wave propagation directions (see Fig. 9) at the three clusters intersect at points that are close to the predicted source coordinates by these two techniques. This, however, is not the case in Plate 2 (see Fig. 10), indicating that Plate 2 exhibits higher degree of anisotropy than Plate 1.

\section{CONCLUSIONS}

Three wave front shape-based acoustic source localization techniques in an anisotropic plate proposed by Park et al. ${ }^{33}$ and Sen and Kundu ${ }^{34}$ have been discussed in this paper. One does not need any a priori knowledge about the material properties in order to employ these techniques. Besides, the techniques do not assume that the acoustic waves propagate in a straight line path from the acoustic source to a sensor. These techniques have been verified previously by Park et al. ${ }^{33}$ and Sen and Kundu ${ }^{34}$ through numerical illustrations. In the present study, an experimental investigation has been preformed to verify the effectiveness of these three techniques in real life applications. The three techniques are based on the analysis of the idealized shapes of the wave fronts typically observed in an anisotropic plate during an acoustic event and then incorporation of the measured signal data by the sensors installed in clusters at several specific locations on the plate. These three techniques are: rhombus ${ }^{33}$, ellipse ${ }^{34}$ and parametric curve ${ }^{34}$-based techniques. A modified version of the rhombus-based approach has been proposed in this study in order to estimate the speed of the wave fronts more realistically than in Park et al. ${ }^{33}$. This modified version requires three sensor clusters instead of four in Park et al. ${ }^{33}$.

The experimental study has been carried out on two plates with different degrees of anisotropy. It has been observed that all the three approaches can predict the source location with sufficient accuracy. For Plate 1, the parametric curve-based approach gives the best source localization result, whereas for Plate 2, the rhombus-based approach gives the best result. The ellipse and parametric-curve based approaches should ideally use the time of arrival information of the second wave groups. However, in this study, the measured signal data do not show an obvious time instant for the second wave group arrival and so the first wave group arrival information has been used even for the ellipse and parametric curve-based approaches. This, however, still gives satisfactory source predictions indicating the robustness of the wave front shapebased techniques.

There is further scope of research in future to properly obtain the second wave arrival information so as to utilize them in the ellipse and parametric curve-based approaches. Also, more sensor clusters can be installed in addition to the three clusters in this paper and different combinations of different numbers of sensor clusters can be considered to experimentally verify the effectiveness of the ellipse and parametric curve-based approaches more extensively. 


\section{REFERENCES}

[1] Tobias, A., "Acoustic emission source location in two dimensions by an array of three sensors", Non-destruct. Test. 9(1), 9-12 (1976).

[2] Kundu, T., "Acoustic source localization", Ultrasonics 54(1), 25-38 (2014).

[3] McLaskey, G. C., Glaser, S. D. and Grosse, C. U., "Beamforming array techniques for acoustic emission monitoring of large concrete structures", J. Sound Vib. 329(12), 2384-2394 (2010).

[4] He, T., Pan, Q., Liu, Y., Liu, X. and Hu, D., "Near-field beamforming analysis for acoustic emission source localization", Ultrasonics 52(5), 587-592 (2012).

[5] Matt, H. M. and Lanza di Scalea, F., "Macro-fiber composite piezoelectric rosettes for acoustic source location in complex structures", Smart. Mater. Struct. 16, 1489-1499 (2007).

[6] Salamone, S., Bartoli, I., di Leo, P., Lanza di Scalea, F., Ajovalasit, A., D’Acquisto, L., Rhymer, J. and Kim, H., "High-velocity impact location on aircraft panels using macrofiber composite piezoelectric rosettes", J. Intel. Mat. Syst. Str. 21, 887-896 (2010).

[7] Salamone, S., Bartoli, I., Rhymer, J., di Scalea, F. L. and Kim, H., "Validation of the Piezoelectric Rosette Technique for Locating Impacts in Complex Aerospace Panels", in: T. Kundu (Ed.), Health Monitoring of Structural and Biological Systems. Pub. Bellingham, Wash, 18thAnnual International Symposium on Smart Structures/NDE, vol. 7984, March 710, 2011, San Diego, California, 2011, pp. 79841E1-11.

[8] Betz, D. C., Thursby, G., Culshaw, B. and Staszewski, W., "Structural damage location with fiber Bragg grating rosettes and lamb waves", Struct. Health Monit. 6(4), 299-308 (2007).

[9] Jiao, J., He, C., Wu, B., Fei, R. and Wang, X., "Application of wavelet transform on modal acoustic emission source location in thin plates with one sensor", Int. J. Pres. Ves. Pip. 81(5), 427-431 (2004).

[10] Surgeon, M. and Wevers, M., "One sensor linear location of acoustic emission events using plate wave theories", Mater. Sci. Eng. A265, 254-261 (1999).

[11] Toyama, N., Koo, J. H., Oishi, R., Enoki, M. and Kishi, T., "Two-dimensional AE source location with two sensors in thin CFRP plates", J. Mater. Sci. Lett. 20(19), 1823-1825 (2001).

[12] Ebrahimkhanlou, A. and Salamone, S., "Acoustic emission source localization in thin metallic plates: A singlesensor approach based on multimodal edge reflections", Ultrasonics 78, 134-145 (2017).

[13] He, T., Xie, Y., Shan, Y., Liu, X., "Localizing Two Acoustic Emission Sources Simultaneously Using Beamforming and Singular Value Decomposition", Ultrasonics 85, 3-22 (2017).

[14] Nakatani, H., Hajzargarbashi, T., Ito, K., Kundu, T. and Takeda, N., "Impact localization on a cylindrical plate by near-field beamforming analysis", in: M. Tomizuka (Ed.), Sensors and Smart Structures Technologies for Civil, Mechanical, and Aerospace Systems, SPIE's 2012 Annual International Symposium on Smart Structures and Nondestructive Evaluation, vol. 8345, San Diego, California, March 12-15, 2012, 2012.

[15] Nakatani, H., Hajzargarbashi, T., Ito, K., Kundu, T. and Takeda, N., "Locating point of impact on an anisotropic cylindrical surface using acoustic beamforming technique", in: 4th Asia-Pacific Workshop on Structural Health Monitoring, Melbourne, Australia, December 5-7, 2012.

[16] Kundu, T., Das, S. and Jata, K. V., "Point of impact prediction in isotropic and anisotropic plates from the acoustic emission data", J. Acoust. Soc. Am. 122(4), 2057-2066 (2007).

[17] Kundu, T., Das, S., Martin, S. A. and Jata, K. V., "Locating point of impact in anisotropic fiber reinforced composite plates", Ultrasonics 48(3), 193-201 (2008).

[18] Hajzargerbashi, T., Kundu, T. and Bland, S., "An improved algorithm for detecting point of impact in anisotropic inhomogeneous plates", Ultrasonics 51(3), 317-324 (2011).

[19] Kundu, T., "A new technique for acoustic source localization in an anisotropic plate without knowing its material properties", in: 6th European Workshop on Structural Health Monitoring, Dresden, Germany, July 3-6, 2012.

[20] Kundu, T., Nakatani, H. and Takeda, N., "Acoustic source localization in anisotropic plates", Ultrasonics 52(6), 740-746 (2012).

[21] Yin, S., Cui, Z. and Kundu, T., "Acoustic source localization in anisotropic plates with "Z" shaped sensor clusters", Ultrasonics 84, 34-37 (2018).

[22] Ciampa, F. and Meo, M., "A new algorithm for acoustic emission localization and flexural group velocity determination in anisotropic structures", Composites Part A 41, 1777-1786 (2010).

[23] Ciampa, F., Meo, M. and Barbieri, E., "Impact localization in composite structures of arbitrary cross section", Struct. Health Monit.: Int. J. 11, 643-655 (2012). 
[24] Kundu, T., Yang, X., Nakatani, H. and Takeda, N., "A two-step hybrid technique for accurately localizing acoustic source in anisotropic structures without knowing their material properties", Ultrasonics 56, 271-278 (2015).

[25] Simone, M. E. D., Ciampa, F., Boccardi, S. and Meo, M., "Impact source localisation in aerospace composite structures", Smart Mater. Struct. 26(12), 125026 (2017).

[26] Sabzevari, S. A. H. and Moavenian, M., "Sound localization in an anisotropic plate using electret microphones", Ultrasonics 73, 114-124 (2017).

[27] Xiao, D., He, T., Pan, Q., Liu, X., Wang, J., Shan, Y., "A novel acoustic emission beamforming method with two uniform linear arrays on plate-like structures", Ultrasonics 54, 737-745 (2014).

[28] Jang, B.-W. and Kim, C.-G., "Impact localization of composite stiffened panel with triangulation method using normalized magnitudes of fiber optic sensor signals", Compos. Struct., 211, 522-529 (2019).

[29] Park, W. H., "Acoustic source localization in an anisotropic plate without knowing its material properties", PhD dissertation, Department of Aerospace and Mechanical Engineering, The University of Arizona, 2016.

[30] Baxter, M. G., Pullin, R., Holford, K. M. and Evans, S. L., "Delta T source location for acoustic emission", Mech. Syst. Signal Process. 21, 1512-1520 (2007).

[31] Hensman, J., Mills, R., Pierce, S. G., Worden, K. and Eaton, M., "Locating acoustic emission sources in complex structures using gaussian processes", Mech. Syst. Signal Process. 24, 211-223 (2010).

[32] Gollob, S., Kocur, G. K., Schumacher, T., Mhamdi, L. and Vogel, T., "A novel multi-segment path analysis based on a heterogeneous velocity model for the localization of acoustic emission sources in complex propagation media", Ultrasonics 74, 48-61 (2017).

[33] Park, W. H., Packo, P. and Kundu, T., "Acoustic source localization in an anisotropic plate without knowing its material properties - A new approach”, Ultrasonics 79, 9-17 (2017).

[34] Sen, N. and Kundu, T., "A new wave front shape-based approach for acoustic source localization in an anisotropic plate without knowing its material properties", Ultrasonics 87, 20-32 (2018).

[35] Nelder, J. A. and Mead, R., “A simplex method for function minimization”, Comput. J. 7, 308-315 (1965).

[36] Lagarias, J. C., Reeds, J. A., Wright, M. H. and Wright, P. E., "Convergence properties of the Nelder-Mead simplex method in low dimensions", SIAM J. Optim. 9 (1), 112-147 (1998). 